\title{
Complete Radiologic Response of Bulky Cerebral Metastases From Newly Diagnosed HER2-Positive Breast Cancer to Upfront Trastuzumab-Based Chemotherapy
}

\author{
Daniel Brungs ${ }^{\mathrm{a}}$, Victor Sze ${ }^{\mathrm{a}}$, Louise Emmett ${ }^{\mathrm{b}}$, Richard J. Epstein ${ }^{\mathrm{a}, \mathrm{c}}$
}

\begin{abstract}
The blood-brain barrier is traditionally regarded as an insurmountable obstacle to the effective drug therapy of brain metastases from solid tumors. Here we describe a striking case of complete radiologic response to chemotherapy, and propose that the critical success factors include the large tumor size, HER2-positivity, and concomitant use of trastuzumab.
\end{abstract}

Keywords: Cerebral metastasis; Blood-brain barrier; Cancer chemotherapy; Breast neoplasms

\section{Introduction}

Oncology practice guidelines teach that neither cytotoxic therapy nor large-molecule target-specific therapies are routinely useful in treating cerebral metastases $[1,2]$ unless these arise from exquisitely chemosensitive primary tumors such as lymphomas or germ-cell neoplasms [3]. To address this unmet need, cerebral metastasectomy and/or sterotactic radiotherapy have become standards of care for most solid tumor patients with intracranial and limited extracranial metastatic disease [4], reflecting perceptions of a blood-brain barrier (BBB) that hinders transmembrane passage of drug molecules larger than $400 \mathrm{Da}$ [5].

In recent years this orthodoxy has been challenged on empirical and theoretical grounds [6], with one objection

Manuscript accepted for publication February 14, 2013

${ }^{a}$ Departments of Oncology, St Vincent's Hospital, Sydney, Australia

${ }^{\mathrm{b}}$ Medical Imaging, St Vincent's Hospital, Sydney, Australia

${ }^{\mathrm{c} C}$ Corresponding author: Richard J. Epstein, The Kinghorn Cancer

Centre, 370 Victoria St, Darlinghurst, Sydney 2010 Australia.

Email: repstein@stvincents.com.au

doi: http://dx.doi.org/10.4021/wjon639w being that the BBB is disrupted by intracerebral deposits larger than $1 \mathrm{~cm}$ [7]. HER2-overexpressing breast cancers are relevant to this debate, given that they recur preferentially within the brain [8], presumably reflecting the rich supply of HER-stimulatory heregulin ligands (neuregulins) [9] within the metastatic 'soil' of the central nervous system [10]. Relevant to this debate, we report here the history of a 50-year-old woman who presented with aggressive metastatic HER2+ breast cancer that required immediate upfront systemic therapy.

\section{Case Report}

A 50-year-old female presented in July 2012 after noticing thickening in the lateral aspect of the right breast. Core biopsy confirmed a $20 \mathrm{~mm}$ hormone receptor-negative grade 2 invasive ductal carcinoma with high levels of HER 2 by both immunohistochemistry and in-situ hybridization. The patient reported no symptoms of metastatic disease, including no headaches or focal symptoms. Examination demonstrated a palpable breast mass with matted axillary lymph nodes, and normal neurological status. PET/CT scan demonstrated FDG-avid metastases in the right breast, right axillary lymph nodes, and multiple bilateral pulmonary nodules; in addition, however, a large photopenic and hypoattenuating area was detected in the right frontal lobe. CT-guided biopsy of a pulmonary nodule confirmed adenocarcinoma. Brain MRI confirmed two frontal lobe lesions (Fig. 1) (upper panels), the largest $28 \mathrm{~mm}$, with extensive surrounding oedema. Gamma-knife surgery was deemed unfeasible due, but this was deemed unfeasible due to the size of the two lesions. Treatment was commenced using dexamethasone for cerebral oedema and 3-weekly standard chemotherapy using docetaxel/carboplatin plus trastuzumab (Herceptin ${ }^{\mathrm{TM}}$; DCH) with pegfilgrastim support. Repeat CT scan prior to the second cycle of $\mathrm{DCH}$ revealed a partial response, while a repeat MRI after five cycles showed a complete response (Fig. 1). There was also a clinical remission of the breast mass, a radiologic complete remission of the axillary adenopathy, plus near-complete remission of the pulmonary metastases. The patient continues on trastuzumab. 


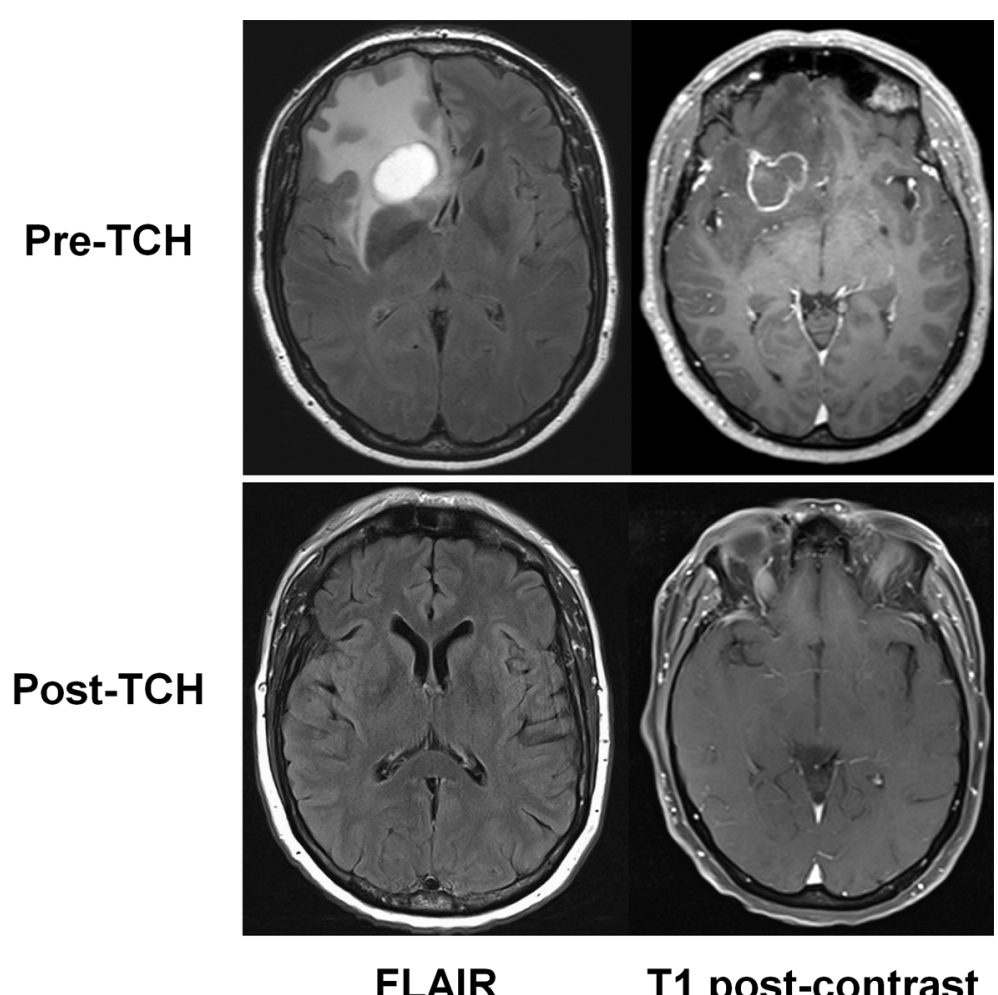

Figure 1. MRI appearances of brain metastases before (pre- $\mathrm{TCH}$, above) and after drug treatment (post-TCH, below). Fluid attenuated inversion recovery (FLAIR) images are shown at left, and T1weighted images at right.

\section{Discussion}

Complete responses of brain metastases to systemic therapies have been reported for kinase inhibitor therapy of lung cancer [11]; for cytotoxic chemotherapy of germ-cell tumors [12]; and in breast cancer, for hormonal therapy [13], concurrent chemoradiation plus lapatinib [14], and (for small brain secondaries only) oral capecitabine [15]. In addition, responses of leptomeningeal breast cancer have been reported using intrathecal trastuzumab [16]. Partial responses of intracerebral metastases are reported in pretreated patients receiving tamoxifen [17], trastuzumab-radiation [18], trastuzumab-cytotoxic combinations [19], and capecitabine-lapatinib [20]. These testimonials to drug efficacy suggest that the traditional notion of the blood-brain barrier may be declining in relevance to the management of brain metastasis.

Trastuzumab reportedly exhibits poor penetration into the brain [21], and is widely believed to be ineffective in controlling brain metastases in breast cancer patients [22]. Despite this, trastuzumab induces radiosensitization in the context of cerebral metastases [23], and patients with HER2positive brain metastases who continue trastuzumab experience longer survival [24, 25]. In contrast, the anticipated efficacy of the small-molecule HER2 kinase inhibitor lapatinib as single-agent therapy for brain metastases has proven to be marginal [26]. Indeed, based on published reports, we can infer no inverse relationship between drug molecular weight (Table 1) and clinical efficacy of brain metastasis therapy in breast cancer patients.

In summary, just as there is growing enthusiasm for first-line use of HER2 inhibitors in lieu of chemotherapy for early breast cancer [27], so does the present case suggest that trastuzumab-based chemotherapy could come to displace surgery and/or radiation therapy in selected cases of HER2-positive bulky brain metastases. Accordingly, in this palliative context, we submit that chemonaive HER2positive intracerebral disease should now join lymphomas and germ-cell tumors in the category of "highly chemosensitive tumors" [3].

\section{Acknowledgement}

DB, VS and RJE acknowledge support from Prof Allan Spigelman and the St Vincents \& Mater Health Cancer Programme.

\section{Disclosures}

All authors declare no conflict of interest. 
Table 1. Molecular Weights of Relevant Oncology Drugs

\begin{tabular}{lll}
\hline Drug & $\begin{array}{l}\text { Reported efficacy in solid tumour } \\
\text { cerebral metastases }\end{array}$ & MWt (Da) \\
\hline Capecitabine & + & 360 \\
Carboplatin & + & 371 \\
Tamoxifen & + & 372 \\
Megestrol acetate & + & 384 \\
Gefitinib & + & 447 \\
Doxorubicin & - & 580 \\
Etoposide & + & 587 \\
Docetaxel & - & 808 \\
Lapatinib & + & 944 \\
Trastuzumab & + & 148,000 \\
\hline
\end{tabular}

The putative blood-brain barrier cut-off is $400 \mathrm{Da}$. MWt: molecular weight; Da: daltons.

\section{Authors' Contributions}

DB and VS collected and interpreted the clinical data, LE supervised the imaging studies and interpretation, DB and RJE conceived and wrote the paper, and all authors were involved in the revision and approval of the final manuscript.

\section{Abbreviations}

BBB: blood-brain barrier; FLAIR: fluid-attenuated image recovery.

\section{References}

1. Lockman PR, Mittapalli RK, Taskar KS, Rudraraju V, Gril B, Bohn KA, Adkins CE, et al. Heterogeneous blood-tumor barrier permeability determines drug efficacy in experimental brain metastases of breast cancer. Clin Cancer Res. 2010;16(23):5664-5678.

2. Lampson LA. Monoclonal antibodies in neuro-oncology: Getting past the blood-brain barrier. MAbs. 2011;3(2):153-160

3. Soffietti R, Cornu P, Delattre JY, Grant R, Graus F, Grisold W, Heimans J, et al. EFNS Guidelines on diagnosis and treatment of brain metastases: report of an EFNS Task Force. Eur J Neurol. 2006;13(7):674-681.

4. Kalkanis SN, Kondziolka D, Gaspar LE, Burri SH, Asher AL, Cobbs CS, Ammirati M, et al. The role of surgical resection in the management of newly diagnosed brain metastases: a systematic review and evidence-based clinical practice guideline. J Neurooncol. 2010;96(1):3343.

5. Pardridge WM. Drug transport across the blood-brain barrier. J Cereb Blood Flow Metab. 2012;32(11):19591972.

6. Fagerholm U. The highly permeable blood-brain barrier: an evaluation of current opinions about brain uptake capacity. Drug Discov Today. 2007;12(23-24):1076-1082.

7. Grimm SA. Treatment of brain metastases: chemotherapy. Curr Oncol Rep. 2012;14(1):85-90.

8. Van Pelt AE, Mohsin S, Elledge RM, Hilsenbeck SG, Gutierrez MC, Lucci A, Jr., Kalidas M, et al. Neoadjuvant trastuzumab and docetaxel in breast cancer: preliminary results. Clin Breast Cancer. 2003;4(5):348-353.

9. Gregory CW, Whang YE, McCall W, Fei X, Liu Y, Ponguta LA, French FS, et al. Heregulin-induced activation of HER2 and HER3 increases androgen receptor transactivation and CWR-R1 human recurrent prostate cancer cell growth. Clin Cancer Res. 2005;11(5):17041712 .

10. Bernstein HG, Lendeckel U, Bertram I, Bukowska A, Kanakis D, Dobrowolny H, Stauch R, et al. Localization of neuregulin-1alpha (heregulin-alpha) and one of its receptors, ErbB-4 tyrosine kinase, in developing and adult human brain. Brain Res Bull. 2006;69(5):546-559.

11. Muller F, Riesenberg H, Hirnle P, Gehl HB, Duwel P, Gorner M. [Complete remission of multiple brain metastases of non-small cell lung cancer induced by gefitinib monotherapy]. Strahlenther Onkol. 2011;187(12):826830.

12. Ohno M, Narita Y. A case of pathological complete remission of a brain metastasis from germ cell tumor of 
the testis after systemic chemotherapy. Jpn J Clin Oncol. 2010;40(12): 1201 .

13. van der Gaast A, Alexieva-Figusch J, Vecht C, Verweij J, Stoter G. Complete remission of a brain metastasis to third-line hormonal treatment with megestrol acetate. Am J Clin Oncol. 1990;13(6):507-509.

14. Abboud M, Saghir NS, Salame J, Geara FB. Complete response of brain metastases from breast cancer overexpressing Her-2/neu to radiation and concurrent Lapatinib and Capecitabine. Breast J. 2010;16(6):644-646.

15. Siegelmann-Danieli N, Stein M, Bar-Ziv J. Complete response of brain metastases originating in breast cancer to capecitabine therapy. Isr Med Assoc J. 2003;5(11):833834.

16. Oliveira M, Braga S, Passos-Coelho JL, Fonseca R, Oliveira J. Complete response in HER2+ leptomeningeal carcinomatosis from breast cancer with intrathecal trastuzumab. Breast Cancer Res Treat. 2011;127(3):841844.

17. Pors H, von Eyben FE, Sorensen OS, Larsen M. Longterm remission of multiple brain metastases with tamoxifen. J Neurooncol. 1991;10(2):173-177.

18. Chargari C, Idrissi HR, Pierga JY, Bollet MA, Dieras V, Campana F, Cottu P, et al. Preliminary results of whole brain radiotherapy with concurrent trastuzumab for treatment of brain metastases in breast cancer patients. Int J Radiat Oncol Biol Phys. 2011;81(3):631-636.

19. Church DN, Bahl A, Jones A, Price CG. HER2-positive breast cancer brain metastases: multiple responses to systemic chemotherapy and trastuzumab--a case report. J Neurooncol. 2006;79(3):289-292.

20. Ro J, Park S, Kim S, Kim TY, Im YH, Rha SY, Chung JS, et al. Clinical outcomes of HER2-positive metastatic breast cancer patients with brain metastasis treated with lapatinib and capecitabine: an open-label expanded access study in Korea. BMC Cancer. 2012;12:322.

21. Stemmler HJ, Schmitt M, Willems A, Bernhard H, Harbeck N, Heinemann V. Ratio of trastuzumab levels in serum and cerebrospinal fluid is altered in HER2-positive breast cancer patients with brain metastases and impairment of blood-brain barrier. Anticancer Drugs. 2007;18(1):23-28.

22. Tosoni A, Franceschi E, Brandes AA. Chemotherapy in breast cancer patients with brain metastases: have new chemotherapic agents changed the clinical outcome? Crit Rev Oncol Hematol. 2008;68(3):212-221.

23. Altundag K, Altundag O, Atik MA, Morandi P, Gunduz M. Rationale for the use of trastuzumab in patients with cerebral metastases who previously receive trastuzumab-based therapy for metastatic breast cancer. Breast. 2005;14(5):425.

24. Xu Z, Marko NF, Chao ST, Angelov L, Vogelbaum MA, Suh JH, Barnett GH, et al. Relationship between HER2 status and prognosis in women with brain metastases from breast cancer. Int J Radiat Oncol Biol Phys. 2012;82(5):e739-747.

25. Pienkowski T, Zielinski CC. Trastuzumab treatment in patients with breast cancer and metastatic CNS disease. Ann Oncol. 2010;21(5):917-924.

26. Toi M, Iwata H, Fujiwara Y, Ito Y, Nakamura S, Tokuda Y, Taguchi T, et al. Lapatinib monotherapy in patients with relapsed, advanced, or metastatic breast cancer: efficacy, safety, and biomarker results from Japanese patients phase II studies. Br J Cancer. 2009;101(10):16761682.

27. Constantinidou A, Smith I. Is there a case for anti-HER2 therapy without chemotherapy in early breast cancer? Breast. 2011;20(Suppl 3):S158-161. 